\title{
A generalization of the Gołab-Schinzel functional equation
}

\author{
JANUSZ MATKOWSKI
}

Dedicated to Professor Janos Aczel on the occasion of his 85th birthday

\begin{abstract}
For every fixed real $p$, the continuous real valued functions $f$ defined on a linear topological space and satisfying the functional equation

$$
f(p[f(y) x+y]+(1-p)[f(x) y+x])=f(x) f(y)
$$

are determined. For $p=0$ or $p=1$ this equation coincides with the classical Gołąb-Schinzel equation.
\end{abstract}

Mathematics Subject Classification (2010). Primary 39B12.

Keywords. Composite equation, gołąb-Schinzel equation, iterative equation, linear topological space, continuous solution.

\section{Introduction}

The functional equation

$$
f(x+y f(x))=f(x) f(y), \quad x, y \in X,
$$

where $X$ is a real linear space and $f: X \rightarrow \mathbb{R}$ an unknown function is the well-known Gołąb-Schinzel equation (cf. [8] also Aczél [1], Aczél [2, pp 132-135], Aczél and Dhombres [3, Chapter 19], Brillouet and Dhombres [6], Javor [9], Wołodźko [12] and Baron [5] where the complex-valued solutions are considered). This equation arises in the problem to determine all subsemigroups of a semigroup which have a faithful continuous parametrization ([4], also [3, Chapter 19]) and, as it contains the superpositions of the unknown functions, it is of the composite type. There are several papers devoted to some generalizations of the (G-S) equation, cf. a survey paper Brzdek [7] and a recent paper by Mureńko [11].

Let $X=\mathbb{R}$. Suppose that $f: X \rightarrow \mathbb{R}$ is an injective solution of the (G-S) equation, and $p: X \times X \rightarrow \mathbb{R}$ is an arbitrary function. Then, obviously, 
$x+y f(x)=f^{-1}(f(x) f(y)), \quad y+x f(y)=f^{-1}(f(x) f(y)), \quad(x, y \in X)$.

Multiplying these equations by $1-p(x, y)$ and $p(x, y)$, respectively, and then adding the resulting equations by sides we get

$$
p[y+x f(y)]+(1-p)[x+y f(x)]=f^{-1}(f(x) f(y)), \quad(x, y \in X),
$$

which implies that $f$ satisfies the functional equation

$$
f(p(x, y)[f(y) x+y]+(1-p(x, y))[f(x) y+x])=f(x) f(y), \quad x \in X .(\mathrm{P})
$$

In this paper we consider the following special case of equation $(\mathrm{P})$ :

$$
f(p[f(y) x+y]+(1-p)[f(x) y+x])=f(x) f(y), \quad x \in X,
$$

where $p \in \mathbb{R}$ is fixed, $X$ is a real linear space and $f: X \rightarrow \mathbb{R}$ an unknown function. For $p=0$ or $p=1$ it becomes the Golab-Schinzel equation. For $p=\frac{1}{2}$ this equation is symmetric with respect to $x$ and $y$, contrary to the (G-S) equation. According to my best knowledge, Eq. 1 is a new generalization of Eq. G-S.

Theorem 1 gives all the continuous solutions $f: \mathbb{R} \rightarrow \mathbb{R}$ of Eq. 1. Since the method of periodic functions (applied in the case of the Golab-Schinzel equation) does not work in the case when $0 \neq p \neq 1$, an essential part of the proof is based on an iterative functional equation (Lemma 2). Applying Theorem 1 we determine the continuous solutions of the form $f: X \rightarrow \mathbb{R}$ where $X$ is a linear topological space (Theorem 2).

At the end of this paper we propose an open problem.

\section{A remark and two lemmas}

The following is easy to verify.

Remark 1. Let $X$ be a real linear space and $p \in \mathbb{R}$ fixed. If a function $f$ : $X \rightarrow \mathbb{R}$ satisfies Eq. 1, then, for every $a \in \mathbb{R}$, the function $X \ni x \rightarrow f(a x)$ also satisfies Eq. 1.

Lemma 1. Let $X$ be a real linear space and $p \in \mathbb{R}$ fixed. If a function $f: X \rightarrow \mathbb{R}$ satisfies Eq. 1 then, for every $k \in \mathbb{N}$,

$$
f\left(\left(\prod_{j=0}^{k-1}\left(1+[f(x)]^{2^{j}}\right)\right) x\right)=[f(x)]^{2^{k}}, \quad x \in X .
$$

Proof. Taking $y=x$ in (1) we see that $f$ satisfies the iterative functional equation

$$
f((1+f(x)) x)=[f(x)]^{2}, \quad x \in X,
$$

so the lemma holds true for $k=1$. Suppose (2) holds true for some $k \in N$. Replacing $x$ by $(1+f(x)) x$ in (2) and using this functional equation we obtain equality (2) for $k+1$ and the induction completes the proof. 
Lemma 2. Let $X$ be a real linear topological space and $p \in \mathbb{R}$ fixed. If a continuous function $f: X \rightarrow \mathbb{R}$ satisfies (1) and $f(X) \cap(0,1) \neq \emptyset$, then $0 \in f(X)$.

Proof. By assumption there exists an $x \in X$ such that $0<f(x)<1$. Since the infinite product $\prod_{j=0}^{\infty}\left(1+[f(x)]^{2^{j}}\right)$ converges, the point

$$
z:=\left(\prod_{j=0}^{\infty}\left(1+[f(x)]^{2^{j}}\right)\right) x
$$

is an element of $X$. By the continuity of $f$ and Lemma 1, we hence get

$$
\begin{aligned}
f(z) & =f\left(\lim _{k \rightarrow \infty}\left(\prod_{j=0}^{k-1}\left(1+[f(x)]^{2^{j}}\right)\right) x\right) \\
& =\lim _{k \rightarrow \infty} f\left(\left(\prod_{j=0}^{k-1}\left(1+[f(x)]^{2^{j}}\right)\right) x\right) \\
& =\lim _{k \rightarrow \infty}[f(x)]^{2^{k}}=0,
\end{aligned}
$$

which completes the proof.

\section{Continuous solutions of real variable}

Theorem 1. Let $p \in \mathbb{R}$ be fixed. A continuous function $f: \mathbb{R} \rightarrow \mathbb{R}$ satisfies the equation

$$
f(p[x f(y)+y]+(1-p)[y f(x)+x])=f(x) f(y), \quad x, y \in \mathbb{R},
$$

if, and only if, either

$$
f(x)=0, \quad x \in \mathbb{R},
$$

or there is $c \in \mathbb{R}$ such that

$$
f(x)=1+c x, \quad x \in \mathbb{R},
$$

or $p \in[0 ; 1]$ and

$$
f(x)=\max (1+c x, 0), \quad x \in \mathbb{R},
$$

for some $c \in \mathbb{R}, c \neq 0$.

Proof. Suppose that a continuous $f: \mathbb{R} \rightarrow \mathbb{R}$ satisfies (3). Setting $x=y=0$ in (3) we get $f(0)=[f(0)]^{2}$ and, consequently, either $f(0)=0$ or $f(0)=1$.

If $f(0)=0$, setting $y=0$ in (3) we obtain $f((1-p) x)=0$ for all $x \in \mathbb{R}$, and setting $x=0$ in $(3)$ we obtain $f(p y)=0$ for all $y \in \mathbb{R}$. It follows that, independently of the value of $p, f(x)=0$ for all $x \in \mathbb{R}$. 
Now assume that $f(0)=1$. Put

$$
Z:=\{x \in \mathbb{R}: f(x)=0\} .
$$

By the continuity of $f$, the set $Z$ is closed.

Part I. We assume that

$$
Z \neq \emptyset \text {. }
$$

If $z_{1}, z_{2} \in Z$ then, by (3),

$$
f\left((1-p) z_{1}+p z_{2}\right)=0,
$$

which proves that $\left((1-p) z_{1}+p z_{2}\right) \in Z$.

Case $p \notin[0 ; 1]$.

If $p<0$ then $1-p>1$. Therefore, Eq. 3 with $p<0$ and Eq. 3 with $p>1$ coincide. Thus, without any loss of generality we can assume that $p>1$.

We shall show that $Z$ defined by (4) is a singleton. For an indirect argument suppose that there are $z_{1}, z_{2} \in Z$ such that $z_{1}<z_{2}$. Then

$$
(1-p) z_{2}+p z_{1}<z_{1}<z_{2}<(1-p) z_{1}+p z_{2}
$$

and

$$
(1-p) z_{2}+p z_{1} \in Z, \quad(1-p) z_{1}+p z_{2} \in Z .
$$

Since $Z$ is closed, it follows that $Z$ is unbounded from both sides.

Hence, taking into account the continuity of $f$ and $f(0)=1$, we conclude that there are $z_{1}, z_{2} \in Z$ such that

$$
z_{1}<0<z_{2} \quad \text { and } \quad f(x)>0 \text { for all } x \in\left(z_{1}, z_{2}\right) .
$$

Setting $x:=z_{k}$ and $y:=x$ in (3) we get

$$
f\left(p\left[z_{k} f(x)+x\right]+(1-p) z_{k}\right)=0, \quad k=1,2, \quad x \in\left[z_{1}, z_{2}\right] .
$$

Since $f(u) \neq 0$ for $u \in\left(z_{1}, z_{2}\right)$, by the continuity of $f$, we infer that, for each $k \in\{1,2\}$, either

$$
p\left[z_{k} f(x)+x\right]+(1-p) z_{k} \leq z_{1}, \quad x \in\left[z_{1}, z_{2}\right] .
$$

or

$$
p\left[z_{k} f(x)+x\right]+(1-p) z_{k} \geq z_{2} \quad x \in\left[z_{1}, z_{2}\right] .
$$

Setting $x=z_{2}$ in inequality (5) we get

$$
p z_{2}+(1-p) z_{k} \leq z_{1}
$$

which is false for $k=1$ and $k=2$. Setting $x=z_{1}$ in (6) we get

$$
p z_{1}+(1-p) z_{k} \geq z_{2}
$$

which is also false for $k=1$ and $k=2$. This contradiction proves that if $p \notin[0,1]$ then $Z$ must be a singleton, that is $Z=\left\{z_{0}\right\}$ for some $z_{0} \neq 0$.

Case $p=1$ or $p=0$. 
Since in each of these two cases Eq. 3 with the classical Gołąb-Schinzel functional equation, it is well known that either $Z=\left\{z_{0}\right\}$ or $Z=\left(-\infty, z_{0}\right]$ or $Z=\left[z_{0}, \infty\right)$.

Case $p \in(0 ; 1)$.

Now $Z$ is $p$-convex. We shall prove that $Z$ is convex. Assume, on the contrary, that it is not true. Then we would find $x, y \in Z, x<y$ such that $[x, y] \backslash Z$ is nonempty. Since $[x, y] \cap Z$ is closed, the set $[x, y] \backslash Z$ is open in $[x, y]$. Consequently, there exist $u, v \in[x, y] \cap Z, u \neq v$, such that $(u, v) \cap Z=\emptyset$. On the other hand, as $u, v \in Z$, from the $p$-convexity of the set $Z$ we have $p u+(1-p) v \in Z \cap A$. This contradiction proves that $Z$ is convex. (For a more general fact see $[10])$.

Taking into account that $0 \notin Z$, one of the following situations must occur:

(i): for some $z_{0}>0$,

$$
Z=\left[z_{0}, \infty\right)
$$

(ii): for some $z_{0}<0$,

$$
Z=\left(-\infty, z_{0}\right]
$$

(iii): for some $z_{0} \in \mathbb{R}, z_{0} \neq 0$,

$$
Z=\left\{z_{0}\right\}
$$

(iv): for some $a, b \in \mathbb{R}, b<a<0$,

$$
Z=[b, a]
$$

(v): for some $a, b \in \mathbb{R}, 0<a<b$,

$$
Z=[a, b] .
$$

Note that the (iv) and (v) cannot happen. Indeed, if for instance (iv) occurs then, setting $y=a$ in Eq. 3, we infer that, for all $x \in \mathbb{R}$,

$$
b \leq p a+(1-p)[a f(x)+x] \leq a,
$$

whence, for all $x \in \mathbb{R}$,

$$
1-\frac{x}{a} \leq f(x),
$$

and, setting $y=b$ in Eq. 3 we infer that, for all $x \in \mathbb{R}$,

$$
b \leq p b+(1-p)[b f(x)+x] \leq a,
$$

whence, for all $x \in \mathbb{R}$,

$$
f(x) \leq 1-\frac{x}{b} .
$$

Consequently we would have

$$
1-\frac{x}{a} \leq 1-\frac{x}{b}, \quad x \in \mathbb{R},
$$

which is impossible. To exclude (v) we can argue in the same way. 
Thus, we have shown that either $Z=\left\{z_{0}\right\}$ or $Z=\left(-\infty, z_{0}\right]$ or $Z=\left[z_{0}, \infty\right)$

To conclude Part I of the proof assume that $Z=\left\{z_{0}\right\}$.

Setting $y:=z_{0}$ in (3) we obtain

$$
f\left(p z_{0}+(1-p)\left[z_{0} f(x)+x\right]\right)=0, \quad x \in \mathbb{R} .
$$

It follows that

$$
p z_{0}+(1-p)\left[z_{0} f(x)+x\right]=z_{0}, \quad x \in \mathbb{R},
$$

whence

$$
f(x)=1-\frac{x}{z_{0}}, \quad x \in \mathbb{R} .
$$

Assume $Z=\left(-\infty, z_{0}\right]$ for some $z_{0}<0$. In this case, as $f$ satisfies Eq. 3, we infer that:

for all $x, y \in \mathbb{R}, y \leq z_{0}($ as $f(y)=0)$,

$$
p y+(1-p)[y f(x)+x] \leq z_{0},
$$

and, for all $x, y \in\left(z_{0},+\infty\right)$,

$$
p[x f(y)+y]+(1-p)[y f(x)+x]>z_{0} .
$$

Hence, taking $y=z_{0}$ in the first of these inequalities and, letting $y \rightarrow z_{0}$ in the second one and taking into account that $f\left(z_{0}\right)=0$, we obtain, respectively,

$$
(1-p)\left[z_{0} f(x)+x\right] \leq(1-p) z_{0}, \quad x \in \mathbb{R},
$$

and

$$
(1-p)\left[z_{0} f(x)+x\right] \geq(1-p) z_{0}, \quad x>z_{0} .
$$

Hence

$$
z_{0} f(x)+x=z_{0}, \quad x>z_{0},
$$

whence

$$
f(x)=1-\frac{x}{z_{0}}, \quad x>z_{0} .
$$

Thus, we have shown that in the case when $Z=\left(-\infty, z_{0}\right]$ the function $f$ must be of the form

$$
f(x)=\max \left(1-\frac{x}{z_{0}}, 0\right), \quad x \in \mathbb{R} .
$$

By Remark 1 , the case $Z=\left[z_{0},+\infty\right)$ reduces to the previous one.

Part 2. Assume that

$$
Z=\emptyset \text {. }
$$

By Lemma 2 we have $f(x) \geq 1$ for all $x \in \mathbb{R}$. Put

$$
S:=\{x \in \mathbb{R}: f(x)=1\} .
$$


The set $S$ is nonempty as $0 \in S$. If $x, y \in S$ then, by (3),

$$
f(x+y)=f(p[x f(y)+y)]+(1-p)[y f(x)+x])=1,
$$

whence $x+y \in S$ and, consequently, $S$ is an additive semigroup of $\mathbb{R}$.

First consider the case when there exists an $x \in S$ such that $x \neq 0$. Without any loss of generality we can assume that $x>0$ (cf. Remark 1 ). Put

$$
S_{+}:=\{x \in S: x>0\} .
$$

We shall show that $a:=\inf S_{+}=0$. Assume for an indirect argument that $a>0$. Since $n a \in S_{+}$for every $n \in \mathbb{N}$, setting $y=n a$ in (3) we obtain

$$
f(x+n p a+n(1-p) a f(x))=f(x), \quad x \in \mathbb{R}, n \in \mathbb{N} .
$$

This equation implies that $f([n a,(n+1) a])=f([0, a])$ for every $n \in N$. Putting

$$
M=\sup f([0 ; \infty))
$$

we infer that there exists an $x_{0} \in[0 ; a]$ such that $f\left(x_{0}\right)=M$. Setting $x=y=$ $x_{0}$ in (3) we get

$$
\left.f\left(x_{0} f\left(x_{0}\right)+x_{0}\right)\right)=\left[f\left(x_{0}\right)\right]^{2}=M^{2} .
$$

Since $M \geq 1$, from the definition of $M$ we conclude that $M^{2} \leq M$ and, consequently, $M=1$. Thus $S_{+}=(0, \infty)$ and, obviously, $a=0$ which is a desired contradiction. This fact easily implies that $S$ is dense in $[0, \infty)$, and, by the continuity of the function $f$,

$$
f(x)=1, \quad x \geq 0 .
$$

Now, setting $y \geq 0$ in (3) and taking into account that $f(y)=1$, we obtain

$$
f(x+p y+(1-p) y f(x))=f(x), \quad y \in[0, \infty), x \in \mathbb{R} .
$$

We can assume without any loss of generality that $1-p>0$. Let $x<0$ be arbitrarily fixed. Since $f(x) \geq 1$, taking $y:=-x$ we have

$$
x+p y+(1-p) y f(x) \geq x+p y+(1-p) y=0,
$$

whence

$$
f(x)=f(x+p y+(1-p) y f(x))=1, \quad x<0 .
$$

Assume that $S=\{0\}$. By Lemma 2 we would have

$$
f(x)>1 \text { for all } x \in \mathbb{R}, x \neq 0 .
$$

To show that this case cannot occur put

$$
F(x, y):=p[x f(y)+y]+(1-p)[y f(x)+x], \quad x, y \in \mathbb{R} .
$$

and observe that, by (3),

$$
F(x, y)=0 \Longleftrightarrow x=y=0 .
$$


Since the function $F$ is continuous in $\mathbb{R}^{2}$ and $f(0)=1$ we infer that $F$ would be positive in $\mathbb{R}^{2} \backslash\{(0,0)\}$. This is impossible as $F(0, y)=y$ for all $y \in \mathbb{R}$. So the case $S=\{0\}$ cannot occur.

This concludes the proof.

Remark 2. It is easy to verify, that every continuous solution $f$ of Eq. 3 satisfies the following conditional functional equation

$$
f(x) \neq 0 \Longrightarrow f\left(-\frac{x}{f(x)}\right) f(x)=1, \quad x \in \mathbb{R} .
$$

Replacing $x$ by $y$ and $y$ by $-\frac{x}{f(x)}$ in (3) and using this relation we obtain

$$
f(x) \neq 0 \Longrightarrow f\left(\frac{p(y-x)+(1-p)(y f(x)-x f(y)}{f(x)}\right)=\frac{f(y)}{f(x)}, \quad x, y \in \mathbb{R} .
$$

Remark 3. Let $p \in \mathbb{R}$ be fixed. If a function $f: \mathbb{R} \rightarrow \mathbb{R}$ is one to one and satisfies Eq. 3 then, for some $c \in \mathbb{R}, c \neq 0$,

$$
f(x)=c x+1, \quad x \in \mathbb{R} .
$$

\section{Main result}

Theorem 2. Let $X$ be a real linear topological space and $p \in \mathbb{R}$ be fixed. $A$ continuous function $f: X \rightarrow \mathbb{R}$ satisfies Eq. 1:

$$
f(p[f(y) x+y]+(1-p)[f(x) y+x])=f(x) f(y), \quad x \in X,
$$

if, and only if, either

$$
f(x)=0, \quad x \in X,
$$

or there is an $x^{*} \in X^{*}$ such that

$$
f(x)=1+<x, x^{*}>, \quad x \in \mathbb{R},
$$

or $p \in[0 ; 1]$ and there exists an $x^{*} \in X^{*} \backslash\{0\}$ such that

$$
f(x)=\sup \left(1+<x, x^{*}>, 0\right), \quad x \in \mathbb{R} .
$$

Proof. Setting $y=0$ in (1) we deduce that either $f(0)=1$ or $f \equiv 0$. The latter is $(7)$. From now on suppose that $f(0)=1$.

Take arbitrarily $x_{0} \in X, x_{0} \neq 0$, and define $g: \mathbb{R} \rightarrow \mathbb{R}$ by

$$
g(t):=f\left(t x_{0}\right), \quad t \in \mathbb{R} .
$$

From (1) we have, for all $s, t \in \mathbb{R}$,

$$
\begin{aligned}
g & (p[s g(t)+t]+(1-p)[t g(s)+s]) \\
& =f\left(\left(p\left[s f\left(t x_{0}\right)+t\right]+(1-p)\left[t f\left(s x_{0}\right)+s\right]\right) x_{0}\right) \\
& =f\left(p\left[f\left(t x_{0}\right) s x_{0}+t x_{0}\right]+(1-p)\left[f\left(s x_{0}\right) t x_{0}+s x_{0}\right]\right)=f\left(s x_{0}\right) f\left(t x_{0}\right) \\
& =g(s) g(t) .
\end{aligned}
$$


Since $g$ is continuous, in view of Theorem 1 , either, for some $c \in \mathbb{R}$, we have

$$
g(t)=1+c t, \quad t \in \mathbb{R},
$$

or $p \in[0,1]$ and, for some $c \in \mathbb{R}, c \neq 0$,

$$
g(t)=\sup (1+c t, 0), \quad t \in \mathbb{R} .
$$

Define $Y:=\{x \in X: f(x)=1\}$. Of course $0 \in Y$ and, by (1), if $x, y \in Y$ then $x+y \in Y$. If $x_{0} \in Y$, using $g$ defined by (10), we infer that $g(0)=g(1)=1$. This fact excludes form (12) for $g$ as, in the opposite case, we would have $1=g(1)=\sup (1+c, 0)$ whence $c=0$. Thus $g$ must be of the form (11) with $c=0$, and, consequently, $g(t)=1$ for all $t \in \mathbb{R}$. It follows that $t x_{0} \in Y$ for all $t \in \mathbb{R}$. We thus have proved that $Y$ is a linear subspace of $X$. The continuity of $f$ implies that $Y$ is closed. Suppose that there are two linearly independent vectors $x_{1}, x_{2} \in X$ such that

$$
\left\{s x_{1}+t x_{2}: s, t \in \mathbb{R}\right\} \cap Y=\{0\} .
$$

Using (10) with $x_{0}$ replaced by $x_{1}$ and $x_{2}$, respectively, we define $g_{1}, g_{2}$ : $\mathbb{R} \rightarrow \mathbb{R}$. Since $x_{1}, x_{2} \notin Y$, according to what we have already shown, these functions are of forms (11) or (12) with $c \neq 0$. It follows that there exist $t_{1}, t_{2} \in \mathbb{R}$ such that $g_{1}\left(t_{1}\right)<1$ and $g_{2}\left(t_{2}\right)>1$. Consider the segment with endpoints $t_{1} x_{1}$ and $t_{2} x_{2}$ :

$$
\left[t_{1} x_{1}, t_{2} x_{2}\right]:=\left\{t\left(t_{1} x_{1}\right)+(1-t) t_{2} x_{2}: t \in[0,1]\right\} .
$$

Since each segment is a connected set and

$$
f\left(t_{1} x_{1}\right)=g_{1}\left(t_{1}\right)<1<g_{2}\left(t_{2}\right)=f\left(t_{2} x_{2}\right),
$$

the Darboux property of $f$ restricted to $\left[t_{1} x_{1}, t_{2} x_{2}\right]$ implies the existence of a number $t \in(0 ; 1)$ such that $f\left(t\left(t_{1} x_{1}\right)+(1-t) t_{2} x_{2}\right)=1$ and, moreover, $t\left(t_{1} x_{1}\right)+(1-t) t_{2} x_{2} \neq 0$. This contradicts (13). Therefore, either $Y=X$, yielding (8) with $x^{*}=0$ or $Y$ is a proper closed hyperplane of $X$. In this last case there exists $y^{*} \in X^{*} \backslash\{0\}$ such that $Y=\left\{x \in X:\left\langle x, x^{*}\right\rangle=0\right\}$. Take $x_{0} \in X \backslash Y$ and put

$$
<x_{0}, x^{*}>=c .
$$

Note that for any $x \in X$ there are unique $t \in \mathbb{R}$ and $u \in Y$ such that $x=$ $u+t x_{0}$. For $x:=t x_{0}$ and $y:=u$ in (1), taking into account that $f(u)=1$, we hence get

$$
\left.f\left(\left[(1-p) f\left(t x_{0}\right)+p\right] u+t x_{0}\right]\right)=f\left(t x_{0}\right), \quad u \in Y, t \in \mathbb{R} .
$$

If the function $g$ given by (10) is of form (12) then $p \in[0,1]$ and, as $g$ is non-negative, we infer that $(1-p) f\left(t x_{0}\right)+p \neq 0$ for all $t \in \mathbb{R}$ when $p \neq 0$. Replacing $u$ by $\frac{u}{(1-p) f\left(t x_{0}\right)+p}$ in (15) we get

$$
f\left(u+t x_{0}\right)=f\left(t x_{0}\right), \quad u \in Y, t \in \mathbb{R} .
$$


If $p=0$ we obtain (16) by putting $x=u, y=t x_{0}$ in (1). Thus, for every $x \in X$ we have $x=u+t x_{0}$ and

$$
f(x)=g(t)=\sup (1+c t, 0) .
$$

Since, by (14),

$$
c t=<x_{0}, x^{*}>t=<t x_{0}, x^{*}>=<u+t x_{0}, x^{*}>=<x, x^{*}>,
$$

we hence get

$$
f(x)=\sup \left(1+<x, x^{*}>, 0\right), \quad x \in X,
$$

that is $f$ is of form $(9)$.

Suppose that the function $g$ is of form (11). Since Eq. 1 with $p=0$ and Eq. 1 with $p=1$ coincide, we can assume without any loss of generality, that $p \neq 1$. Simple calculation shows that

$$
(1-p) f\left(t x_{0}\right)+p=(1-p) g(t)+p=0 \quad \text { iff } \quad t=\frac{1}{(p-1) c} .
$$

Therefore, replacing $u$ by $\frac{u}{(1-p) f\left(t x_{0}\right)+p}$ in (15) we get

$$
f\left(u+t x_{0}\right)=f\left(t x_{0}\right), \quad u \in Y, t \in \mathbb{R} \backslash\left\{\frac{1}{(p-1) c}\right\} .
$$

The continuity of $f$ implies that in this case (16) also holds true. Now, similarly as in the previous case, for every $x \in X$, we have

$$
f(x)=g(t)=1+c t=1+<x, x^{*}>,
$$

that is $f$ is of form (8).

To show the "if" part of the theorem take arbitrary $x, y \in X$. Since $x=u+s x_{0}$ and $y=v+t x_{0}$ for some (uniquely determined) $u, v \in Y$ and $s, t \in \mathbb{R}$, it is easy to see that functions (7), (8) and (9) satisfy Eq. 1 iff the real variable function $g$ defined by (10) satisfies Eq. 3. An application of Theorem 1 completes the proof.

Remark 4. In the proof of the above theorem we apply a modified method which is due to N. Brillouet and J. Dhombres [6] (cf. also [3]).

Remark 5. Let $X$ be a real linear space, $h: X \rightarrow \mathbb{Q}$ an arbitrary additive function, and $p \in \mathbb{Q}$ where $\mathbb{Q}$ denotes the set of rational numbers. Then the function $f: X \rightarrow \mathbb{Q}$ given by

$$
f(x)=1+h(x), \quad x \in \mathbb{R},
$$

is a solution of Eq. 3 ([2, pp. 134-135]). 
Indeed, making use of the additivity of $h$ and, repeatedly, the rational homogeneity of additive functions, we have, for all $x, y \in X$,

$$
\begin{aligned}
f & (p[x f(y)+y]+(1-p)[y f(x)+x]) \\
& =h(p(x[h(y)+1]+y)+(1-p)(y[h(x)+1]+x))+1 \\
& =p h(x[h(y)+1])+p h(y)+(1-p) h(y[h(x)+1])+(1-p) h(x)+1 \\
& =p[h(y)+1] h(x)+p h(y)+(1-p)[h(x)+1] h(y)+(1-p) h(x)+1 \\
& =h(x) h(y)+h(x)+h(y)+1=[h(x)+1][h(y)+1]=f(x) f(y) .
\end{aligned}
$$

Of course, if $h$ is not identically zero, the function $f$ in this remark is discontinuous everywhere.

We end the paper with the following open

Problem 1. Determine all (or all continuous) functions $f$ and $p$ satisfying Eq. P.

Open Access. This article is distributed under the terms of the Creative Commons Attribution Noncommercial License which permits any noncommercial use, distribution, and reproduction in any medium, provided the original author(s) and source are credited.

\section{References}

[1] Aczél, J.: Beirtäge zur Theorie der geometrischen Objecte III-IV. Acta Math. Acad. Sci. Hungar. 8, 19-52 (1957)

[2] Aczél, J.: Lectures on Functional Equations and their Applications. Cambridge University Press, Academic Press, New York (1966)

[3] Aczél, J., Dhombres, J.: Functional Equations in Several Variables, Encyclopedia of Mathematics and its Applications. Cambridge University Press, Cambridge (1989)

[4] Aczél, J., Gołąb, St.: Remarks on one-parameter subsemigroups of the affine group and their homo- and isomorphisms. Aequationes Math. 4, 1-10 (1970)

[5] Baron, K.: On the continuous solutions of the Gołąb-Schinzel equation. Aequationes Math. 38, 155-162 (1989)

[6] Brillouet, N., Dhombres, J.: Equations fonctionelleset recherche de sous-groupes. Aequationes Math. 31, 253-293 (1986)

[7] Brzdęk, J.: The Gołąb-Schinzel equation and its generalizations. Aequationes Math. 70, 14-24 (1970)

[8] Gołąb, St., Schinzel, A.: Sur l'équation fonctionnelle $f[x+y f(x)]=f(x) f(y)$. Publ. Math. Debrecen 6, 113-125 (1960)

[9] Javor, P.: On the general solution of the functional equation $f[x+y f(x)]=f(x) f(y)$. Aequationes Math. 1, 235-238 (1968)

[10] Matkowski, J., Ślepak, W.: On $(\alpha, a)$-convex set valued functions. Far East J. Math. Sci. 4(1), 85-89 (2002)

[11] Mureńko, A.: On the general solution of a generalization of the Goląb-Schinzel equation. Aequationes Math. 77, 107-118 (2009)

[12] Wołodźko, S.: Solution générale de l'équation fonctionelle $f[x+y f(x)]=f(x) f(y)$. Aequationes Math. 2, 12-29 (1968) 
Janusz Matkowski

Faculty of Mathematics

Computer Sciences and Econometrics

University of Zielona Góra

Podgórna 50

65246 Zielona Góra

Poland

Janusz Matkowski

Institute of Mathematics

Silesian University

Bankowa 14

40007 Katowice

Poland

e-mail: J.Matkowski@wmie.uz.zgora.pl

Received: May 27, 2010

Revised: August 5, 2010 